\title{
SAR Imaging Exploiting Multi-path
}

\author{
V. Krishnan ${ }^{1}$, C. E. Yarman ${ }^{2}$, Member, IEEE, and B. Yazıc ${ }^{1}$, Senior Member, IEEE \\ ${ }^{1}$ Department of Electrical, Computer and Systems Engineering \\ Rensselaer Polytechnic Institute \\ 110, 8th Street, Troy, NY 12180 \\ Phone: (518)-276 2905, Fax: (518)-276 6261 \\ ${ }^{2}$ Houston Technology Center, WesternGeco-Schlumberger, Houston, TX 77042 \\ E-mail: V. K. - krishv2@rpi.edu, C. E. Y. - yarman@ecse.rpi.edu and B. Y. - yazici@ecse.rpi.edu
}

\begin{abstract}
We present a new filtered-backprojection (FBP) type image reconstruction method for synthetic aperture radar (SAR) that exploits multi-path in the presence of a perfectly reflecting wall. The new method is designed to take advantage of multiple scattering and incorporates the multiple scattered waves in the image reconstruction process enhancing the reconstructed image. We present numerical simulation to demonstrate the performance of the new method. While the focus of this paper is on radar applications, our image formation method is also applicable to other problems arising in fields such as acoustic, geophysical and medical imaging.
\end{abstract}

\section{INTRODUCTION}

In SAR, a scene of interest is illuminated by electromagnetic (EM) waves transmitted from an airborne platform, such as an airplane or satellite. Then an image of the scene is reconstructed from the measurement of the back-scattered EM waves. In reconstruction of the image, a common assumption made about the medium where EM waves travel before reaching the scene of interest is that the medium is scatterer free. This assumption does not necessarily hold in a scattering rich medium, such as an urban environment with many buildings, where scattering follows multiple paths (multi-path) between the SAR platforms and the scene.

In recent years there have been studies towards understanding and incorporating multi-path scattering in the SAR image reconstruction process. It has been shown that proper incorporation of multi-path information offers potential gains in image reconstruction such as increase in aperture and resolution. In [2], a medium with point-like scatterers was considered and an imaging method that exploited the multi-path between the scene of interest and these point-like scatterers was developed. It was shown that multi-path scattering incorporates views from extra directions. In [3], [7], a vertical perfectly reflecting wall adjacent to the scene of interest was considered. Under the assumption that the receiver platform can isolate the EM waves scattered from different scattering paths, it was shown in [7] that multi-path enhances image resolution. On a follow up work [3], the structure of the artifacts was studied when the isolation of multi-path assumption is dropped.

In this paper, we design an FBP type image reconstruction method that exploits multi-path scattering. Similar to our previous work [5], [13], we use microlocal analysis in a

This work was supported by the Air Force Office of Scientific Research (AFOSR) under the agreement FA9550-07-1-0363 and by the National Science Foundation (NSF) under Grant No. CCF-08030672. statistical setting to design our image reconstruction method. Microlocal techniques lead to inversion methods that have the desirable property that visible edges in the scene will appear in the reconstructed image at the correct location and orientation [4], [10], [11]. They can also account for various factors such as arbitrary flight trajectory, non-flat ground topography, antenna beam pattern, transmitted waveform and geometric spreading. Furthermore, microlocal techniques give rise to FBP type inversion, which is a direct (noniterative) method and can be potentially implemented fast [6].

In this paper, we consider the imaging scenario of [3] where the scene of interest is adjacent to a perfectly reflecting wall, co-located transmitter and receiver on an airborne platform, i.e. monostatic SAR, and the receiver cannot isolate the multipaths. As in [3] we consider the following four possible scattering paths:

(a) Transmitter - target - receiver

(b) Transmitter - wall - target - receiver

(c) Transmitter - target - wall - receiver

(d) Transmitter - wall - target - wall - receiver

These four scattering paths are described schematically in Figure 1.

Given the four different scattering paths above, we treat the multi-path imaging set-up in a multi-static setting involving two transmitters and two receivers, see Equation (4). We employ the techniques introduced in [5], [13] to reconstruct the image of the target while suppressing the artifacts caused by data interference from different scattering paths. As in [5], our method involves backprojecting the received data with respect to each transmitter-receiver pair. We assume that we have $a$ priori knowledge of the second-order statistics of the target to be reconstructed. We determine the second-order statistics of the artifacts caused by interference based on different scattering paths and design a filter that preserves the singularities in the received data due to the scene while suppressing the artifact singularities in a mean-square error (MSE) sense. We form the final image by superposition of images reconstructed for all transmit-receive pairs. The novelty of our work is that the filters we derive in this paper reconstructs the image and suppresses the artifacts, as against the work of [3], which accounts for the artifacts caused in multi-path imaging.

This paper is organized as follows: In Section II, we introduce the multi-path SAR forward model in a statistical setting. In Section III, we introduce the FBP type operators and derive the filters. Numerical simulation is presented in Section 

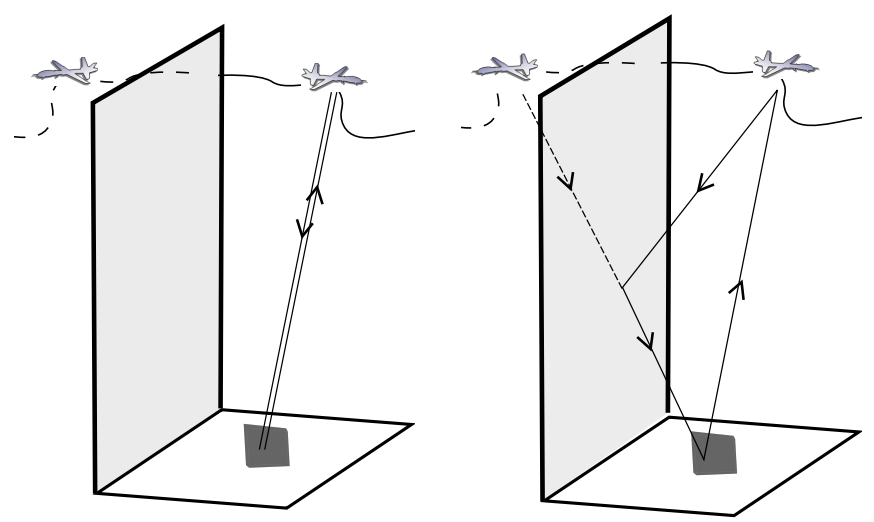

(a)

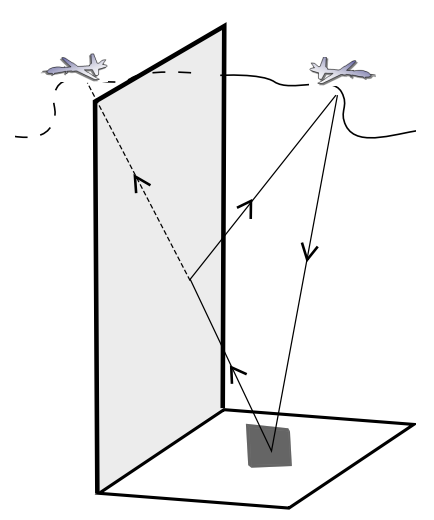

(c) (b)

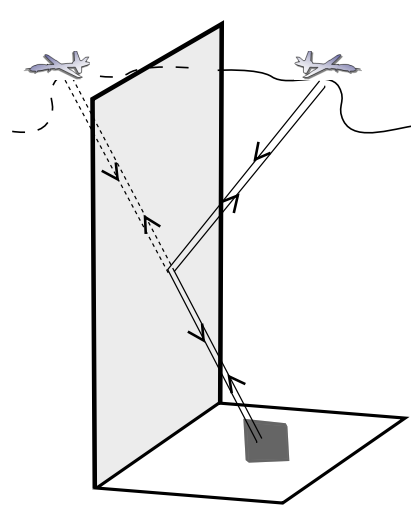

(d)

Fig. 1. The four scattering paths in multi-path SAR set-up.

IV and Section V concludes this paper. The stationary phase method on which our filter derivation is based is presented in Appendix A.

\section{FORWARD MODEL FOR MULTI-PATH SAR}

We use the following notational conventions in this paper. Operators are denoted in calligraphic font, points in $\mathbb{R}^{n}$ for $n \geq 3, \mathbb{R}^{2}$ and $\mathbb{R}$ are denoted in bold Roman, bold italic and italic letters respectively.

We assume a mono-static SAR system traversing the trajectory $\gamma(s)=\left\langle\gamma_{1}(s), \gamma_{2}(s), \gamma_{3}(s)\right\rangle \in \mathbb{R}^{3}$ for $s \in\left[s_{0}, s_{1}\right]$. Let the ground topography be defined by $\mathbf{x}=(\boldsymbol{x}, \psi(\boldsymbol{x}))$ where $\psi: \mathbb{R}^{2} \rightarrow \mathbb{R}$ is a known smooth function for $\boldsymbol{x} \in \mathbb{R}^{2}$. We assume a perfectly reflecting vertical wall adjacent to the scene of interest and denote the orthogonal reflection of $\gamma$ with respect to the wall by $\widetilde{\gamma}$. We assume that EM waves propagate in free-space and are scattered off the wall or in a thin region near the earth's surface. Consequently, we denote the scene reflectivity by $T(\boldsymbol{x})$ for $\boldsymbol{x} \in \mathbb{R}^{2}$ [9].

We assume that $T$ is zero-mean, second order stationary in $\boldsymbol{x}$ whose auto-covariance function $R_{T}$ is given by

$$
\left.R_{T}\left(\boldsymbol{x}, \boldsymbol{x}^{\prime}\right)=\mathrm{E}\left[T(\boldsymbol{x}) \overline{T\left(\boldsymbol{x}^{\prime}\right)}\right)\right] \text { for } \boldsymbol{x}, \boldsymbol{x}^{\prime} \in \mathbb{R}^{2},
$$

where E denotes the statistical expectation operator. Then the power spectral density of $T$ defined by

$$
\widetilde{S}_{T}\left(\zeta, \zeta^{\prime}\right)=\frac{1}{(2 \pi)^{4}} \int e^{\mathrm{i}\left(\boldsymbol{x} \cdot \zeta-\boldsymbol{x}^{\prime} \cdot \zeta^{\prime}\right)} R_{T}\left(\boldsymbol{x}, \boldsymbol{x}^{\prime}\right) \mathrm{d} \boldsymbol{x} \mathrm{d} \boldsymbol{x}^{\prime},
$$

satisfies

$$
\widetilde{S}_{T}\left(\zeta, \zeta^{\prime}\right)=S_{T}(\zeta) \delta\left(\zeta-\zeta^{\prime}\right)
$$

Following [7], we model the received signal $d$ as

$$
d(s, t)=\mathcal{F}[T](s, t):=\left(\mathcal{F}_{1}+\mathcal{F}_{2}+\mathcal{F}_{3}+\mathcal{F}_{4}\right)[T](s, t),
$$

where

$$
\mathcal{F}_{1}[T](s, t)=\int e^{-\mathrm{i} \omega\left(t-\frac{1}{c_{0}} R_{\boldsymbol{\gamma}, \boldsymbol{\gamma}}(\boldsymbol{x}, s)\right)} A_{1}(\boldsymbol{x}, s, \omega) T(\boldsymbol{x}) \mathrm{d} \boldsymbol{x} \mathrm{d} \omega .
$$

$$
\mathcal{F}_{2}[T](s, t)=-\int e^{-\mathrm{i} \omega\left(t-\frac{1}{c_{0}} R_{\boldsymbol{\gamma}, \tilde{\boldsymbol{\gamma}}}(\boldsymbol{x}, s)\right)} A_{2}(\boldsymbol{x}, s, \omega) T(\boldsymbol{x}) \mathrm{d} \boldsymbol{x} \mathrm{d} \omega .
$$$$
\mathcal{F}_{3}[T](s, t)=-\int e^{-\mathrm{i} \omega\left(t-\frac{1}{c_{0}} R_{\boldsymbol{\gamma}, \tilde{\boldsymbol{\gamma}}}(\boldsymbol{x}, s)\right)} A_{3}(\boldsymbol{x}, s, \omega) T(\boldsymbol{x}) \mathrm{d} \boldsymbol{x} \mathrm{d} \omega
$$$$
\mathcal{F}_{4}[T](s, t)=\int e^{-\mathrm{i} \omega\left(t-\frac{1}{c_{0}} R_{\tilde{\boldsymbol{\gamma}}, \tilde{\boldsymbol{\gamma}}}(\boldsymbol{x}, s)\right)} A_{4}(\boldsymbol{x}, s, \omega) T(\boldsymbol{x}) \mathrm{d} \boldsymbol{x} \mathrm{d} \omega .
$$

Here $R_{\boldsymbol{\gamma}, \widetilde{\boldsymbol{\gamma}}}(\boldsymbol{x}, s)=|\boldsymbol{x}-\boldsymbol{\gamma}(s)|+|\boldsymbol{x}-\widetilde{\boldsymbol{\gamma}}(s)|, s \in\left[s_{0}, s_{1}\right]$ is the slow-time variable, $t \in\left[t_{0}, t_{1}\right]$ is the fast-time variable, $c_{0}$ is the speed of light in free air, $\omega$ is the temporal frequency and $A_{i}$ for $i=1, \cdots, 4$ are functions that take into account, the transmitter and receiver antenna beam patterns, transmitted waveforms and geometric spreading factors [1], [8], [9]. We assume that the functions $A_{i}(s, \boldsymbol{x}, \omega)$ for $i=1, \cdots, 4$ satisfy the following estimate for some real number $m_{i}$ :

$$
\begin{aligned}
\left|\partial_{\omega}^{\alpha} \partial_{s}^{\beta} \partial_{x_{1}}^{\rho_{1}} \partial_{x_{2}}^{\rho_{2}} A_{i}(s, \boldsymbol{x}, \omega)\right| & \leq C\left(1+|\omega|^{2}\right)^{\left(m_{i}-|\alpha|\right) / 2} \\
& \text { for }(s, \boldsymbol{x}) \in K \text { and } \omega \in \mathbb{R}
\end{aligned}
$$

where $K \subset \mathbb{R} \times \mathbb{R}^{2}$ is any compact set, $\alpha, \beta, \rho_{1}, \rho_{2}$ are any non-negative integers, $C$ is a constant in terms of $p, q, K, \alpha$, $\beta, \rho_{1}$ and $\rho_{2}$. The assumption (9) is needed to make various stationary phase calculations. It also defines each operator $\mathcal{F}_{i}$ as a Fourier Integral Operator [10], [11]. The assumption (9) is satisfied when the antenna is broadband and the transmitted waveform is band-limited. We assume that the ideal received is not necessarily decomposable into components. Thus the multi-path problem involves reconstructing the reflectivity function of the scene based on the model (4). We develop a FBP type image reconstruction method for the multi-path SAR image reconstruction problem in the next section.

\section{IMAGE RECONSTRUCTION}

We treat the received signal (4) in the multi-path SAR setup as the superposition of the received data from a multistatic SAR set-up involving two transmitters and two receivers traversing trajectories $\gamma$ and $\widetilde{\gamma}$. As a consequence, we use the methods developed in [5], [13] to develop FBP type image reconstruction operators. We design FBP type image reconstruction operator for each transmitter-receiver pair that suppresses artifacts in the reconstructed image due to FBP of the data received from different scattering paths. Designed FBP operators amplifies the strength of the singularities of the 
scene contained within the data collection manifold of a given transmitter-receiver pair while suppressing the singularities of the scene contained within the data collection manifolds of the other transmitter-receiver pairs in a minimum MSE sense.

For each transmitter-receiver pair $(\gamma, \gamma),(\gamma, \widetilde{\gamma}),(\widetilde{\gamma}, \gamma)$ and $(\widetilde{\gamma}, \widetilde{\gamma})$, we design the filters $B_{1}, B_{2}, B_{3}$ and $B_{4}$ corresponding to the FBP operators $\mathcal{B}_{1}, \mathcal{B}_{2}, \mathcal{B}_{3}$ and $\mathcal{B}_{4}$, respectively. Since the derivation of the filters, $B_{1}, B_{3}$ and $B_{4}$ are similar to that of $B_{2}$, we present the derivation of $B_{2}$ following the methods of [5], [13].

We define the FBP operator $\mathcal{B}_{2}$ by

$$
\mathcal{B}_{2}[d](\boldsymbol{z})=\int e^{\mathrm{i} \omega\left(t-\frac{2}{c_{0}} R_{\boldsymbol{\gamma}, \tilde{\boldsymbol{\gamma}}}\right)} B_{2}(\boldsymbol{z}, s, \omega) d(s, t) \mathrm{d} s \mathrm{~d} t \mathrm{~d} \omega,
$$

and corresponding image $\widetilde{T}_{2}$ formed by using $\mathcal{B}_{2}$ by

$$
\widetilde{T}_{2}(\boldsymbol{z})=\mathcal{B}_{2}[d](\boldsymbol{z}) .
$$

Substituting (4) in (11), we obtain

$$
\widetilde{T}_{2}(\boldsymbol{z})=\mathcal{B}_{2} \mathcal{F}_{2}[T](\boldsymbol{z})+\sum_{i=1, i \neq 2}^{4} \mathcal{B}_{2} \mathcal{F}_{i}[T](\boldsymbol{z})
$$

$\mathcal{B}_{2} \mathcal{F}_{2}$ is the familiar bi-static SAR image reconstruction operator presented in [12]. It is a pseudodifferential operator and hence applying this operator to the scene reflectivity function $T$ preserves the edges of $T$ at the right location and orientation in the reconstructed image. On the contrary, $\mathcal{B}_{2} \mathcal{F}_{i}$ are not necessarily pseudodifferential operators, therefore they do not reconstruct the edges of $T$ at the right location. We design $\mathcal{B}_{2}$ such that the singularities of the target in the bi-static data collection manifold corresponding to the pair $(\gamma, \widetilde{\gamma})$ are preserved while the singularities of the target in the data collection manifolds corresponding to the pairs $(\gamma, \gamma)$ and $(\widetilde{\gamma}, \widetilde{\gamma})$ are suppressed in the minimum MSE sense.

The bi-static data collection manifold corresponding to the pair $(\gamma, \widetilde{\gamma})$ is given by

$\left.\Omega_{\boldsymbol{z}}^{\boldsymbol{\gamma}, \widetilde{\boldsymbol{\gamma}}}:=\left\{\frac{\omega}{c_{0}} D\left(\boldsymbol{z}_{1}, \boldsymbol{z}_{2}\right)^{T}(\widehat{\boldsymbol{z}-\boldsymbol{\gamma}(s})+\widehat{\boldsymbol{z}-\widetilde{\gamma}(s)}\right): A_{2}(\boldsymbol{z}, s, \omega) \neq 0\right\}$

with

$$
D\left(\boldsymbol{z}_{1}, \boldsymbol{z}_{2}\right)=\left(\begin{array}{cc}
1 & 0 \\
0 & 1 \\
\frac{\partial \psi}{\partial \boldsymbol{z}_{1}} & \frac{\partial \psi}{\partial \boldsymbol{z}_{2}}
\end{array}\right)
$$

The superscript $T$ denotes transpose of the matrix $D\left(\boldsymbol{z}_{1}, \boldsymbol{z}_{2}\right)$ and $\widehat{\boldsymbol{v}}$ indicates unit vector in the direction $\boldsymbol{v}$. The bi-static data collection manifold determines the best possible image $T_{\Omega_{z}^{\gamma, \tilde{\gamma}}}$ that can be reconstructed by the transmitter-receiver pair $(\gamma, \widetilde{\gamma})[12]:$

$$
T_{\Omega_{\boldsymbol{z}}^{\gamma, \tilde{\gamma}}(\boldsymbol{z}):=} \int_{\Omega_{\boldsymbol{z}}^{\boldsymbol{\gamma}, \tilde{\boldsymbol{\gamma}}}} e^{\mathrm{i}(\boldsymbol{x}-\boldsymbol{z}) \cdot \boldsymbol{\xi}} T(\boldsymbol{x}) \mathrm{d} \boldsymbol{x} \mathrm{d} \boldsymbol{\xi} .
$$

We compute the filter $B_{2}$ such that the MSE

$$
I\left(B_{2}\right)=E\left[\int\left|\widetilde{T}_{2}(\boldsymbol{z})-T_{\Omega_{\boldsymbol{z}}^{\boldsymbol{\gamma}, \tilde{\gamma}}}(\boldsymbol{z})\right|^{2} \mathrm{~d} \boldsymbol{z}\right]
$$

is minimized.
From (16) we derive the filter as follows. First we substitute the integral representations of $\mathcal{B}_{i}$ and $\mathcal{F}_{i}$ in (12) and perform the change of variables

$$
(s, \omega) \rightarrow \boldsymbol{\xi}=\frac{\omega}{c_{0}} \Sigma_{\boldsymbol{\gamma}, \widetilde{\gamma}}(\boldsymbol{x}, \boldsymbol{z}, s)
$$

along with a stationary phase approximation (see Appendix A). Here

$$
\Sigma_{\boldsymbol{\gamma}, \widetilde{\boldsymbol{\gamma}}}(\boldsymbol{x}, \boldsymbol{z}, s)=\int_{0}^{1} \nabla R_{\boldsymbol{\gamma}, \widetilde{\boldsymbol{\gamma}}}(s, \boldsymbol{z}+\lambda(\boldsymbol{x}-\boldsymbol{z})) \mathrm{d} \lambda
$$

with $\nabla$ denoting the gradient operator in the $\boldsymbol{x}$ variable. For $\boldsymbol{x}=\boldsymbol{z}$,

$$
\Sigma_{\boldsymbol{\gamma}, \widetilde{\boldsymbol{\gamma}}}(\boldsymbol{z}, \boldsymbol{z}, s)=\left[D\left(\boldsymbol{z}_{1}, \boldsymbol{z}_{2}\right)\right]^{T}((\widehat{\boldsymbol{\gamma}(s)-\boldsymbol{z}})+(\widetilde{\boldsymbol{\gamma}(s)-\boldsymbol{z}})),
$$

where $D\left(\boldsymbol{z}_{1}, \boldsymbol{z}_{2}\right)$ is as in (14). In other words $\Sigma_{\boldsymbol{\gamma}, \widetilde{\boldsymbol{\gamma}}}(\boldsymbol{z}, \boldsymbol{z}, s)$ is the projection of the bisector of $(\widehat{\gamma(s)-z})$ and $(\widetilde{\gamma(s)-z})$ onto tangent plane at $\boldsymbol{z}$.

Then writing

$$
R_{\boldsymbol{\gamma}, \widetilde{\boldsymbol{\gamma}}}(s, \boldsymbol{x})-R_{\boldsymbol{\gamma}, \widetilde{\gamma}}(s, \boldsymbol{z})=(\boldsymbol{x}-\boldsymbol{z}) \cdot \Sigma_{\boldsymbol{\gamma}, \widetilde{\boldsymbol{\gamma}}}(\boldsymbol{x}, \boldsymbol{z}, s),
$$

and performing stationary phase approximation, we simplify $\mathcal{B}_{2} \mathcal{F}_{2}(\boldsymbol{z})$ as

$$
\begin{array}{r}
\mathcal{B}_{2} \mathcal{F}_{2}(\boldsymbol{z})=\int e^{\mathrm{i}(\boldsymbol{x}-\boldsymbol{z}) \cdot \boldsymbol{\xi}} B_{2}(\boldsymbol{z}, \boldsymbol{\xi}) A_{2}(\boldsymbol{x}, \boldsymbol{\xi})\left|J_{2}(\boldsymbol{x}, \boldsymbol{z}, \boldsymbol{\xi})\right| \\
\times T(\boldsymbol{x}) \mathrm{d} \boldsymbol{x} \mathrm{d} \boldsymbol{\xi} .
\end{array}
$$

For notational brevity we write $A_{2}(\boldsymbol{x}, \boldsymbol{\xi})=A_{2}(\boldsymbol{x}, s(\boldsymbol{\xi}), \omega(\boldsymbol{\xi}))$ and $B_{2}(\boldsymbol{z}, \boldsymbol{\xi})$ is defined similarly.

Using the change of variables (17) on the remaining terms of (12), we obtain,

$$
\begin{array}{r}
\mathcal{B}_{2} \mathcal{F}_{1}(\boldsymbol{z})=\int e^{\mathrm{i}(\boldsymbol{x}-\boldsymbol{z}) \cdot \boldsymbol{\xi}} B_{2}(\boldsymbol{z}, \boldsymbol{\xi}) A_{1}(\boldsymbol{x}, \boldsymbol{\xi})\left|J_{2}(\boldsymbol{x}, \boldsymbol{z}, \boldsymbol{\xi})\right| \\
\times \widetilde{T} 1(\boldsymbol{x}, \boldsymbol{\xi}) \mathrm{d} \boldsymbol{x} \mathrm{d} \boldsymbol{\xi}
\end{array}
$$

where

$$
\widetilde{T}_{1}(\boldsymbol{x}, \boldsymbol{\xi}):=\widetilde{T}_{1}(\boldsymbol{x}, s(\boldsymbol{\xi}), \omega(\boldsymbol{\xi}))=e^{\mathrm{i} \triangle_{1}(\boldsymbol{x}, s(\boldsymbol{\xi}), \omega(\boldsymbol{\xi}))} T(\boldsymbol{x})
$$

and

$$
\triangle_{1}(\boldsymbol{x}, s(\boldsymbol{\xi}), \omega(\boldsymbol{\xi}))=\frac{\omega(\boldsymbol{\xi})}{c_{0}}(|\mathbf{x}-\gamma(s(\boldsymbol{\xi}))|-|\mathbf{x}-\widetilde{\gamma}(s(\boldsymbol{\xi}))|) .
$$

Similarly,

$$
\begin{array}{r}
\mathcal{B}_{2} \mathcal{F}_{4}(\boldsymbol{z})=\int e^{\mathrm{i}(\boldsymbol{x}-\boldsymbol{z}) \cdot \boldsymbol{\xi}} B_{2}(\boldsymbol{z}, \boldsymbol{\xi}) A_{4}(\boldsymbol{x}, \boldsymbol{\xi})\left|J_{2}(\boldsymbol{x}, \boldsymbol{z}, \boldsymbol{\xi})\right| \\
\times \widetilde{T}_{4}(\boldsymbol{x}, \boldsymbol{\xi}) \mathrm{d} \boldsymbol{x} \mathrm{d} \boldsymbol{\xi}
\end{array}
$$

where

$$
\widetilde{T}_{4}(\boldsymbol{x}, \boldsymbol{\xi}):=\widetilde{T}_{1}(\boldsymbol{x}, s(\boldsymbol{\xi}), \omega(\boldsymbol{\xi}))=e^{\mathrm{i} \triangle_{4}(\boldsymbol{x}, s(\boldsymbol{\xi}), \omega(\boldsymbol{\xi}))} T(\boldsymbol{x})
$$

and

$\triangle_{4}(\boldsymbol{x}, s(\boldsymbol{\xi}), \omega(\boldsymbol{\xi}))=\frac{\omega(\boldsymbol{\xi})}{c_{0}}(|\mathbf{x}-\widetilde{\gamma}(s(\boldsymbol{\xi}))|-|\mathbf{x}-\gamma(s(\boldsymbol{\xi}))|)$.

Note that $\mathcal{B}_{2} \mathcal{F}_{3}$ has the same expression as $\mathcal{B}_{2} \mathcal{F}_{2}$. 
Finally substituting (21), (22) and (25) in (16), using the stationary phase theorem and taking the variational derivative of (16) with respect to $B_{2}$, we compute the filter $B_{2}$ as

$$
B_{2}(\boldsymbol{x}, \boldsymbol{\zeta})=\frac{\chi_{\Omega_{z}^{\gamma}, \tilde{\gamma}} \overline{A_{2}(\boldsymbol{x}, \boldsymbol{\zeta})} S_{T}(\boldsymbol{\zeta})}{\Omega}
$$

where

$$
\begin{aligned}
\Omega & =\left|J_{2}(\boldsymbol{x}, \boldsymbol{x}, \boldsymbol{\zeta})\right|\left(\left(\left|A_{2}(\boldsymbol{x}, \boldsymbol{\zeta})\right|^{2}+\left|A_{3}(\boldsymbol{x}, \boldsymbol{\zeta})\right|^{2}\right) S_{T}(\boldsymbol{\zeta})+\right. \\
& \left.+\sum_{i=1, i \neq 2,3}^{4}\left|A_{i}(\boldsymbol{x}, \boldsymbol{\zeta})\right|^{2} S_{T}\left(\boldsymbol{\zeta}+\partial_{x} \triangle_{i}(0, \boldsymbol{\zeta})\right)\right)
\end{aligned}
$$

Here $\chi_{\Omega_{z}^{\gamma}, \tilde{\gamma}}$ is the characteristic function equal to 1 on the data collection manifold (13) and zero everywhere else.

The other filters can be derived similarly. The final image is obtained by superposition.

\section{NUMERICAL SIMULATION}

In our numerical simulations, we considered a scene of size $[0,22] \times[0,22] \mathrm{km}^{2}$ discretized to a $128 \times 128$ grid of pixels with $(0,0)$ and $(22,22)$ corresponding to pixel numbers $(1,1)$ and $(128,128)$ respectively. The scene consists of a circular and an elliptic target as shown in Fig. 2(a). The perfectly reflecting vertical wall is perpendicular to the scene, whose cross section is shown by the blue vertical line in Fig. 2(a). We made the assumptions that the earth's surface is flat and the amplitude functions $A_{i} \equiv 1$ for $i=1, \cdots, 4$. This choice of amplitude functions corresponds to isotropic transmitters and receivers using a delta function as the transmit waveform. The parameters we used correspond to a system bandwidth of approximately $0.873 \mathrm{MHz}$.

The transmitter traverses a half-circular trajectory $\gamma$ of radius $11 \mathrm{~km}$ centered at $(11,11)$ and at a constant height of $6.5 \mathrm{~km}$ above the target scene: $\gamma(s)=\langle 11+11 \cos (s), 11+$ $11 \sin (s), 6.5\rangle$ for $0 \leq s \leq \pi$. The trajectory reflected about the vertical wall is $\widetilde{\gamma}(s)=\langle 11+11 \cos (s), 11-11 \sin (s), 6.5\rangle$ for $0 \leq s \leq \pi$ (See Fig. 2(b)).

We used a discrete version of the multi-path forward data model (Equation (4)) with $A_{i}=1$ for $i=1, \cdots, 4$, to generate our simulation data. In our simulations, shown in Fig. 3, we compared our multi-path reconstruction scheme with the mono-static reconstruction method of [9], [13]. We see that the reconstruction algorithm introduced in this paper is able to reconstruct the image and at the same time reduce the artifacts induced by using the mono-static reconstruction algorithm of [9], [13]. We should point out that our multipath image reconstruction algorithm does induce artifacts of the original scene reflected about cross section of the vertical wall. These cannot be suppressed, but these will appear on the other side of the wall.

\section{CONCLUSion}

In this paper, we developed an FBP type inversion method for multi-path SAR in a mono-static SAR imaging scenario. We assumed that the target to be imaged is near a perfectly reflecting vertical wall and took into account the different scattering paths that interact with the target once. As a result, the signal at the receiver may be interfered by these scattered signals taking different scattering paths. We made the assumption that we have a priori knowledge of the power spectral density function of the target and developed an FBP type imaging algorithm that reconstructs the image of the target while suppressing the artifacts caused by interference due to multiple scattering paths. While our paper has focused primarily on image formation for multi-path SAR, the techniques are also applicable to other imaging problems such as those arising in acoustics, geophysics and tomography.

\section{APPENDIX A}

\section{STATIONARY PHASE METHOD}

Let $u$ be a smooth function of compact support in $\mathbb{R}^{n}$ and let $\varphi$ be a real valued function with only non-degenerate critical points. A point $x_{0} \in \mathbb{R}^{n}$ is called a non-degenerate critical point if $D \varphi\left(x_{0}\right)=0$ and the Hessian matrix $D^{2} \varphi\left(x_{0}\right)$ has non-zero determinant. The stationary phase theorem states as $\lambda \rightarrow \infty$,

$$
\begin{array}{r}
\int e^{\mathrm{i} \lambda \varphi(\boldsymbol{x})} u(\boldsymbol{x}) \mathrm{d} \boldsymbol{x}=\left(\frac{2 \pi}{\lambda}\right)_{\left\{\boldsymbol{x}_{0}: D \varphi\left(\boldsymbol{x}_{0}\right)=0\right\}}^{n / 2} \sum \frac{e^{\mathrm{i} \lambda \varphi\left(\boldsymbol{x}_{0}\right)} e^{\mathrm{i} \pi / 4 \operatorname{sgn} D^{2} \varphi\left(\boldsymbol{x}_{0}\right)}}{\sqrt{\left|\operatorname{det} D^{2} \varphi\left(\boldsymbol{x}_{0}\right)\right|}} \\
+\mathcal{O}\left(\lambda^{-n / 2-1}\right) .
\end{array}
$$

\section{REFERENCES}

[1] M. Cheney, Synthetic-aperture assessment of a dispersive surface, International Journal of Imaging Systems and Technology 14 (2004), 28-34.

[2] M. Cheney and R. J. Bonneau, Imaging that exploits multipath scattering from point scatterers, Inverse Problems 20 (2004), no. 5, 1691-1711.

[3] R. Gaburro and C. J. Nolan, Enhanced imaging from multiply scattered waves, Inverse Probl. Imaging 2 (2008), no. 2, 225-250.

[4] A. Grigis and J. Sjöstrand, Microlocal analysis for differential operators, London Mathematical Society Lecture Note Series, vol. 196, Cambridge University Press, Cambridge, 1994, An introduction.

[5] V. Krishnan, J. Swoboda, C. E. Yarman, and B. Yazıcı, Multi-static synthetic aperture radar image formation, Accepted for publication in IEEE Trans. Image Process.

[6] S. Nilsson, Application of fast backprojection techniques for some inverse problems of integral geometry, Ph.D. thesis, Linkping Studies Sci.Technol., 1997.

[7] C. J. Nolan, M. Cheney, T. Dowling, and R. Gaburro, Enhanced angular resolution from multiply scattered waves, Inverse Problems 22 (2006), no. $5,1817-1834$

[8] C.J. Nolan and M. Cheney, Synthetic aperture inversion, Inverse Problems 18 (2002), 221-236.

[9] __ Synthetic aperture inversion for arbitrary flight paths and nonflat topography, IEEE Transactions on Image Processing 12 (2003), 1035-1043.

[10] F. Trèves, Introduction to pseudodifferential and Fourier integral operators. Vol. 1, Plenum Press, New York, 1980, Pseudodifferential operators, The University Series in Mathematics.

[11] Introduction to pseudodifferential and Fourier integral operators. Vol. 2, Plenum Press, New York, 1980, Fourier integral operators, The University Series in Mathematics.

[12] C. E. Yarman, B. Yazıcı, and M. Cheney, Bistatic synthetic aperture radar imaging for arbitrary flight trajectories, IEEE Trans. Image Process. 17 (2008), no. 1, 84-93.

[13] B. Yazici, M. Cheney, and C.E. Yarman, Synthetic-aperture inversion in the presence of noise and clutter, Inverse Problems 22 (2006), 17051729. 


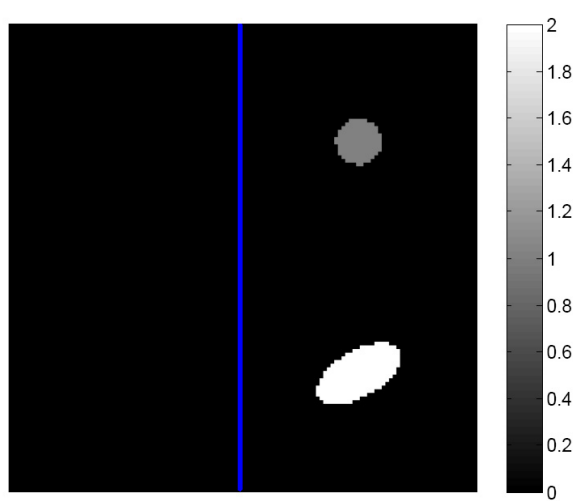

(a)

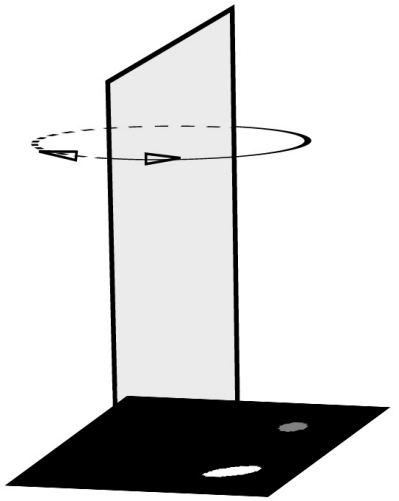

(b)

Fig. 2. (a) Scene used in numerical simulations. The blue vertical line shows the cross section of the wall. (b) 3-dimensional view of the trajectory of the transmitter (solid line) and the reflected trajectory about the vertical wall (dotted line).

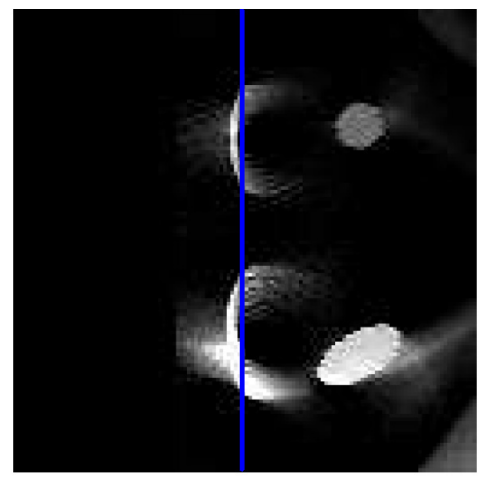

(a)

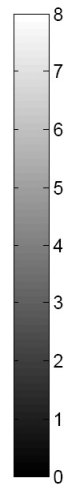

Fig. 3. (a) Image reconstructed by the mono-static FBP image reconstruction algorithm of [9], [13]. (b) Image reconstructed by the multi-path algorithm of this paper

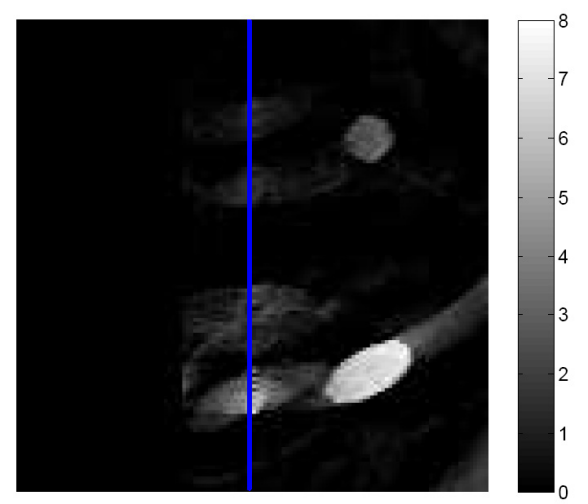

(b) 